\title{
Single-pass measurement of the optical quality of the opossum eye
}

\author{
Eduardo Oswaldo-Cruz ${ }^{1}$, Cristovam W. Picanço-Diniz ${ }^{2}$ and Luiz Carlos L. Silveira² \\ 1 - Universidade Federal do Rio de Janeiro, Rio de Janeiro, RJ, Brazil \\ 2 - Universidade Federal do Pará, Belém, PA, Brazil
}

\begin{abstract}
This paper reports the results that are part of a series of experiments designed to evaluate aspects of the spatial resolution of the visual system of the opossum, Didelphis marsupialis aurita. This nocturnal marsupial presents a well-developed eye, displaying features that reflect specialization for operation at low levels of luminosity. The species was shown to be slightly myopic, a feature that may prove to be valuable because of the increased depth of field. Opossum visual acuity has been previously evaluated by means of determining the Contrast Sensitivity Function (CSF). The results indicate rather poor visual acuity compared with other nocturnal animals. In this paper, we describe the results obtained for the optical quality of the opossum's eye using a single-pass method. The results suggest that the opossum's optical system is capable of forming images that can be resolved when separated by an angular distance on the order of 6 minutes of arc. Keywords: eye optics, eye optical quality, single-pass method, contrast sensitivity, opossum eye.
\end{abstract}

Received 8 June 2011; received in revised form 30 June 2011; accepted 30 June 2011. Available on line 30 June 2011

\section{Introduction}

The visual system provides information about the spatial and temporal distribution of the radiation of a restricted portion of the electromagnetic spectrum from a wide extent of the space that surrounds an animal. The analysis of spatial luminosity distribution plays a leading role in the organization of the behavior of the animal in its ecological niche. In higher vertebrates, spatial analysis is performed on the image of the external world formed over the photoreceptor layer by the refractive elements of the eye. Information provided by the selective activation of these elements is integrated at the peripheral level by a complex network of neural elements that constitute the neural portion of the retina. These preliminary data are then conveyed by means of the nerve fibers that constitute the optic nerve to higher centers where further analysis occurs, resulting in perception.

Acuity is a measure of the ability to discriminate between separate points in space and for a particular

Eduardo Oswaldo-Cruz, Universidade Federal do Rio de Janeiro, Instituto de Biofísica Carlos Chagas Filho, Rio de Janeiro, Brazil. Cristovam W. Picanço-Diniz and Luiz Carlos L. Silveira, Universidade Federal do Pará, Instituto de Ciências Biológicas, Belém, Brazil. Luiz Carlos L. Silveira, Universidade Federal do Pará, Núcleo de Medicina Tropical, Belém, Brazil. Correspondence regarding this article should be directed to: Dr. Luiz Carlos L. Silveira, Universidade Federal do Pará, Núcleo de Medicina Tropical, Av. Generalíssimo Deodoro, no 92 (Umarizal), 66055-240 Belém, Pará, Brazil. Phone: +559132016819. Fax:+5591-32410032. E-mail: luiz@ufpa.br species is determined by three major factors. The first factor concerns the optical quality of the image formed by the eye. The second factor concerns the ability of the neural retina to code and analyze the activity that arises from receptor activation. The third factor is sensitivity. In some species, the ability to resolve fine detail varies with the intensity of the stimuli.

During several years of study, various morphological and functional aspects of the visual system of the opossum, Didelphis marsupialis aurita, a nocturnal marsupial with a wide geographical distribution, were analyzed. This species offers unique opportunities for neurobiological research. For example, its particular mode of reproduction renders possible the study of the early development of the organization of the visual pathway. Marsupials have many features in common with primitive eutherian mammals; therefore, better knowledge of their nervous system may offer important clues to understanding the organization of the brains of higher mammals.

Several topics of the organization of the opossum visual system have been studied, indicating that marsupials have many features in common with higher mammals. To understand the optical and neural factors that determine the spatial resolution of this species, we performed a series of experiments designed to evaluate the limitations set by optical and neural elements that comprise opossum eye optics (Oswaldo Cruz, Hokoç, \& Sousa, 1979), eye refractive state (Picanço-Diniz, Silveira, \& Oswaldo-Cruz, 1983), retinal ganglion cell density distribution (Hokoç \& Oswaldo Cruz, 1979), contrast sensitivity, and visual acuity (Silveira, 
Picanço-Diniz, \& Oswaldo-Cruz, 1982). In this paper, we describe the results obtained for the optical quality of the opossum's eye using a single-pass method. The results were previously published in a symposium book with a very limited number of printed copies (Oswaldo Cruz, Picanço-Diniz, \& Silveira, 1982).

\section{Materials, methods and results}

We estimated the quality of the image formed at the retinal level by the refractive system of the opossum's eye, evaluating the Line Spread Function of its dioptric system. We used a "single-pass" method introduced by Hisako Ikeda and colleagues (Ikeda \& Wright, 1973) to study the optical quality of the retinal image of the eye of the cat.

One adult specimen of Didelphis marsupialis aurita (WIED, 1826) that weighed $1300 \mathrm{~g}$ was used. The animal was anesthetized by an intraperitoneal injection of the barbiturate sodium pentobarbital, $25 \mathrm{mg} / \mathrm{kg}$, and mounted on a head holder. Eye movements were prevented by continuous intravenous infusion of the neuromuscular blocking agent pancuronium bromide (Pavulon, $10 \mathrm{mg} / \mathrm{kg} / \mathrm{h}$; Organon, São Paulo, Brazil). The animal was artificially ventilated. Cycloplegia, midriasis, and exophthalmia were induced by the topical application of atropine and phenilephrine. Body temperature was maintained by a thermostatically controlled electric blanket. Electrocardiographic monitoring was conducted throughout the experiment. All photographic recordings of the fundus occuli were performed under anesthesia.

After an intravenous injection of a $10 \%$ solution of fluoresceine, the retinal vessels of both animal eyes were photographed using a fundus camera (Carl Zeiss Jena, Jena, East Germany). The negatives were developed using a gamma value of approximately 1 . The negatives were then placed in an apparatus that consisted of a high-intensity light source, condenser, and photographic objective. A rotary front surface mirror driven by a triangular waveform generator displaced the enlarged image across a slit in front of a photodetector. The profile of the smallest capillary vessels was then measured by means of the output of a photomultiplier coupled to a PDP-12 digital computer (Digital Equipment Corporation, Maynard, MA, USA) and interactive software that allowed the averaging of the photomultiplier output (Zin \& Oswaldo Cruz, 1981). Figure 1 is a semi-diagrammatic representation of the procedure used to obtain these data. We determined the half-width at mid-height of the luminosity profiles of the smallest vessels $(n>20)$ present in the fundus occuli photographic recordings. The results were in the range of 6-12 minutes of arc and suggest that the optical system is capable of forming images that can be resolved when separated by an angular distance on the order of 6 minutes of arc. Therefore, the optical quality of the image formed by the refractive system of the opossum's eye is almost as good as that formed by the eyes of cats and humans (i.e., 1-2 minutes of arc; Campbell \& Gubisch, 1966; Ikeda \& Wright, 1973; Ikeda, Wright, Young, \& Nuza, 1973; Vos, Walraven, \& Van Meeteren, 1976; Westheimer, 1986; Williams, Brainard, McMahon, \& Navarro, 1994).

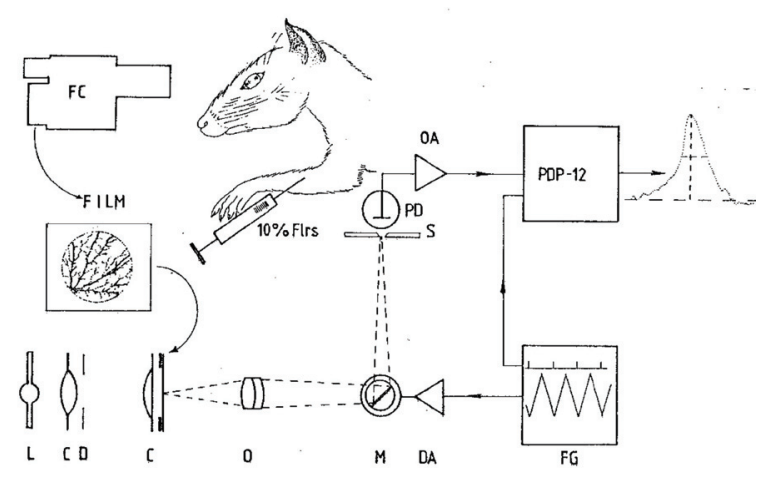

Figure 1. Semi-diagrammatic representation of the experimental procedures used for the determination of the optical quality of the retinal image. A Zeiss Jena fundus camera was used to photograph the retinal vessels following an intravenous injection of a $10 \%$ solution of fluoresceine. After developing (gamma approximately 1) the negatives, they were placed in an apparatus that consisted of a high-intensity light source, condenser, and photographic objective. A rotary front surface mirror driven by a triangular waveform generator displaced the enlarged image across a slit in front of a photodetector. After amplification, the output of the sensor was averaged using an interactive program in a PDP-12 digital computer. The luminance profile of the smallest vessels was displayed and photographed. Measurements to determine the half-width at mid-height were performed on prints or directly in the calibrated scope screen.

\section{Discussion}

This analysis of the quality of the retinal image does not take into consideration the refractive state of the eye (Oswaldo-Cruz et al., 1979; Picanço-Diniz et al., 1983). Knowledge of the optical properties of the eye of the opossum was obtained by computing the schematic eye for this species using Gaussian simplification (OswaldoCruz et al., 1979). The results suggest that this species shows some degree of myopia (-1.97 D), contrasting with the hyperopia usually reported in small eyes when slit retinoscopy is used. The results obtained by Gaussian calculation are valid for para-axial rays in aberrationfree systems. The small eye of this species (AP diameter approximately $10 \mathrm{~mm}$ ), displaying high lightgathering properties, presents chromatic and spherical aberrations as expected (unpublished observations). In an aberration-free system, resolution is a function of both the wavelength of light and aperture of the system, but in practice this limit is not attained because of the 
limitations introduced by the imperfection of refractive components. Picanço-Diniz et al. (1983) used visually evoked cortical potentials to study 25 animals and estimated that this species shows an average myopia of $-2.21 \mathrm{D}$, giving strong experimental support to the myopic refractive state of the opossum's eye.

Silveira et al. (1982) used visually evoked cortical potentials to estimate the contrast sensitivity and visual acuity of the opossum in a study of 11 animals. They found that average visual acuity in this sample was 1.25 cycles per degree, indicating that the opossum has lower visual acuity than primates, felines, and squirrels but higher visual acuity than albino and pigmented rats. The acuity obtained in the mesopic range $\left(2.4 \mathrm{~cd} / \mathrm{m}^{2}\right)$ was not improved when stimuli of considerably higher luminance $\left(500-1000 \mathrm{~cd} / \mathrm{m}^{2}\right)$ were used, suggesting that the retina of this species does not present a higherresolution system that operates at photopic levels.

Low contrast sensitivity, poor spatial resolution, and the absence of a dual system that operates at different luminosity levels offer further evidence that this species is endowed with a visual apparatus specialized to operate at low luminosity.

\section{Acknowledgements}

EOC, CWPD, and LCLS have received continuous support from CNPq, CAPES, and FINEP during their careers.

\section{References}

Campbell, F.W., \& Gubish, R.W. (1966). Optical quality of human eye. Journal of Physiology (London), 186, 558-578.

Hokoç, J.N., \& Oswaldo Cruz, E. (1979). A regional specialization in the opossum's retina: quantitative analysis of the ganglion cell layer. Journal of Comparative Neurology, 183, 385-395.

Ikeda, H., \& Wright, M.J. (1973) Optical quality of the cat's eye and human eye. Journal of Physiology (London), 232, 34P-35P.

Ikeda, H., Wright, M.J., Young, S., \& Nuza, J. (1973). Relation between refractive error and the spread of the image on the cat's retina. Vision Research, 13, 867-871.

Oswaldo-Cruz, E., Hokoç, J.N., \& Sousa, A.P.B. (1979). A schematic eye for the opossum. Vision Research, 19, 263-278.

Oswaldo-Cruz, E., Picanço-Diniz, C.W., \& Silveira, L.C.L. (1982). Optical and neural factors involved in spatial resolution by the visual system of the opossum Didelphis marsupialis. Proceedings of the Third Japan-Brazil Symposium on Science and Technology, (pp. 147-161). Tokyo.

Picanço-Diniz, C.W., Silveira, L.C.L., \& Oswaldo-Cruz, E. (1983). Electrophysiological determination of the refractive state of the eye of the opossum. Vision Research, 23, 867-872.

Silveira, L.C.L., Picanço-Diniz, C.W., \& Oswaldo-Cruz, E. (1982). Contrast sensitivity function and visual acuity of the opossum. Vision Research, 22, 1371-1377.

Vos, J.J., Walraven, J., \& Van Meeteren, A. (1976). Light profiles of the foveal image of a point source. Vision Research, 16, 215-219.

Westheimer, G. (1986). The eye as an optical instrument. In K.R. Boff, L. Kaufman, \& J.P. Thomas (Eds.), Sensory processes and perception (series title: Handbook of perception and human performance, vol. 1) (pp. 4/1-4/20). New York, N.Y.: Wiley.

Williams, D.R., Brainard, D.H., McMahon, M.J., \& Navarro, R. (1994). Double-pass and interferometric measures of the optical quality of the eye. Journal of the Optical Society of America: A. Optics, image science, and vision, 11, 3123-3135.

Zin, W.A., \& Oswaldo Cruz, E. (1981). AVG: a signal averager for biological data. International Journal of Biomedical Computing, $12,149-156$ 